\title{
INTERPRÉTATION GÉOMÉTRIQUE DES INTÉGRALES DE FRESNEL;
}

Par M. N. OUMOFF.

I. - Pour représenter géométriquement les intégrales de Fresnel :

$$
\mathrm{A}=\int_{0}^{5} \cos \frac{\pi}{2} z^{2} d z, \quad \mathrm{~B}=\int_{0}^{5} \sin \frac{\pi}{2} z^{2} d z
$$

enroulons la parabole:

$$
\frac{\pi}{2} z^{2}=v
$$

sur un cylindre circulaire droit d'axe $o z$ ( $f g .1$ ), de manière que le sommet de la parabole tombe sur $o x$ et que l'axe $o v$ de la parabole

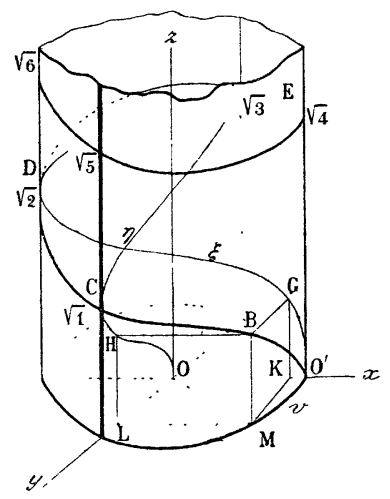

FIG. 1.

s'enroule sur la circonférence de base du cylindre dans le plan $x y$.

La parabole (1) s'enroule alors suivant deux hélices, l'une ascendante, l'autre descendante, qui correspondent à ses deux branches. Il suffit de considérer, par exemple, la branche ascendante représentée dans la fig. 1: un point $\mathrm{H}$ de cette branche a pour coordonnées :

$$
\begin{gathered}
x=\mathrm{OK}=\cos v, \quad y=\mathrm{OL}=\sin v, \\
z=\mathrm{MB}=\mathrm{LH}=\mathrm{KG}=\sqrt{\frac{2}{\pi} v} .
\end{gathered}
$$

J. de phys., $3^{\circ}$ série, t. VI. (Juin 1897.)

21 
Considérons les projections de cette hélice sur le plan $x z$, courbe $\xi$ ( $f g .1$ et $f g .2)$, et sur le plan $y z$, courbe $\eta(f i g .1$ et $f i g .3)$.

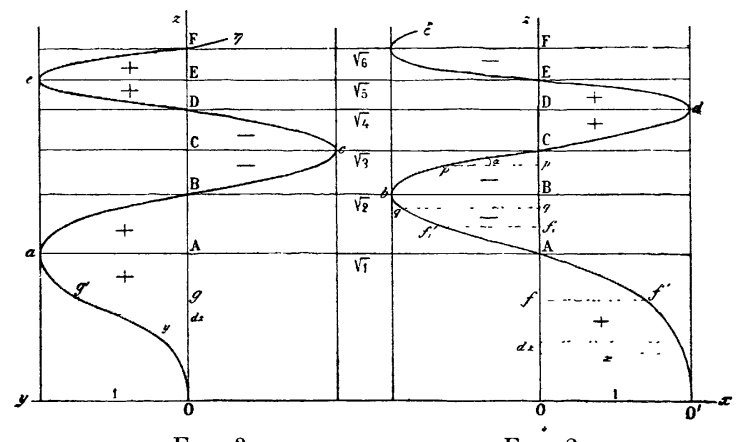

Fı. 3.

FIG. 2.

La courbe $\xi$ coupe $o z$ aux points définis par :

$$
v=(2 h+1) \frac{\pi}{2} \text {, c'est-à-dire } z_{\xi}=\sqrt{2 h+1} \text { (où } h=0,1,2,3, \ldots \text {. }
$$

La courbe $\eta$ coupe $o z$ aux points tels que:

$$
v=2 h \frac{\pi}{2} \quad \text { et } \quad z_{n}=\sqrt{2 h} .
$$

C'est ce qu'on voit sur les fig. 2 et 3 . On voit aussi que $\xi$ oscille dans le plan zox entre les génératrices du cylindre qu'elle touche en ses sommets successifs ; la variation d'ordonnée d'un sommet à l'autre tend vers zéro et les branches de courbe se rapprochent rapidement des tronçons d'une ligne brisée.

II. - L'aire comprise entre la courbe $\xi$, l'axe des $z$ (fig. 2) et les ordonnées $o o^{\prime}, f f^{\prime}$, a pour valeur :

$$
\int_{0}^{z} x d z=\int_{0}^{z} \cos \frac{\pi}{2} z^{2} d z
$$

Elle représente toujours l'intégrale A de Fresnel si l'on compte les boucles de $\xi$ limitées à $o z$ comme positives du côté des $x$ positifs, et comme négatives du côté des $x$ négatifs $(f i g .2)$.

Formée ainsi par une somme algébrique d'aires alternativement positives et négatives, l'intégrale $\mathrm{A}$ est maximum après chaque 
boucle positive, c'est-à-dire pour la limite supérieure $\mathrm{Z}=\sqrt{2 h+1}$ définie par $v=(4 h+1) \frac{\pi}{2}$, et A est minimum après chaque boucle négative, c'est-à-dire pour

$$
\mathrm{Z}=\sqrt{4 h+3}, \quad v=(4 h-1) \frac{\pi}{2}
$$

De même la courbe $\eta$ (fig. 3) représente l'intégrale B de Fresnel sous des conditions analogues.

Les maximum de $B$ sont définis par:

$$
\mathrm{Z}=\sqrt{2(2 h+1)}, \quad v=2(2 h+1) \frac{\pi}{2}
$$

et les minịmum, par:

$$
\mathrm{Z}=\sqrt{4 h,} \quad v=4 h \frac{\pi}{2} .
$$

Ce sont bien là les résultats connus qu'on retrouve ici par une méthode nouvelle.

III. - Formule approchée pour le calcul des intégrales de Fresnel entre deux limites d'intégration assez rapprochées.

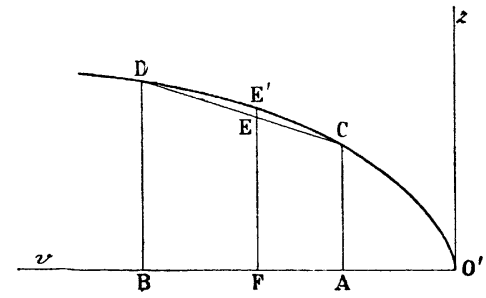

FIG. 4.

Soient C et D (fig. 4$)$ deux points de la parabole (1).

En posant:

$$
\mathrm{AG}=z_{0}, \quad \mathrm{BD}=z_{1}, \quad \mathrm{FE}=\zeta, \quad \mathrm{AB}=\alpha, \quad 0^{\prime} \mathrm{A}=v_{0}, \quad 0^{\prime} \mathrm{F}=v
$$

on a:

$$
\zeta=\frac{z_{1}-z_{0}}{\alpha}\left(v-v_{0}\right)+z_{0} ; \quad d \zeta=\frac{z_{1}-z_{0}}{\alpha} d v
$$


Si $C$ et $D$ sont assez rapprochés, on peut remplacer $E^{\prime} F$ par $E F$, el $d z$ par $d \zeta$, alors:

$\mathrm{A}_{z_{1}}^{z_{1}}=\int_{0}^{v_{1}} \cos v d \zeta=\frac{z_{1}-z_{0}}{\alpha} \int_{0}^{v_{1}} \cos v d v=\frac{z_{1}-z_{0}}{\alpha}\left[\sin \frac{\pi}{2} z_{1}^{2}-\sin \frac{\pi}{2} z_{0}^{2}\right]$

Maintenant :

$$
\alpha=\frac{\pi}{2}\left(z_{1}^{2}-z_{0}^{2}\right)
$$

Par suite :

De même :

$$
\left.\begin{array}{l}
\mathrm{A}_{z_{0}}^{z_{1}}=\frac{2}{\pi} \frac{\sin \frac{\pi}{2} z_{1}^{2}-\sin \frac{\pi}{2} z_{0}^{2}}{z_{1}+z_{0}} \\
\mathbf{B}_{z_{0}}^{z_{1}}=-\frac{2}{\pi} \frac{\cos \frac{\pi}{2} z_{1}^{2}-\cos \frac{\pi}{2} z_{0}^{2}}{z_{1}+z_{0}}
\end{array}\right\}
$$

Par exemple, Fresnel trouve $\mathrm{A}_{0}^{12}=0,7161$. En posant: $z_{1}=1,3$ et $z_{0}=1,2$, on a: $\mathrm{A}_{0}^{13}=0,6390 ;$ Fresnel donne : 0,6393.

IV. - Calcul approché des intégrales de Fresnel entre deux limites quelconques. - On y arrive en remplaçant les courbes $\xi$ et, $\eta$ par des lignes convenables plus simples.

$1^{\circ}$ Premier quart de spire de l'hélice. -- Intégrale A. - Le premier quart despire $o^{\prime} f^{\prime} \mathrm{A}$ de $\xi(f i g .2)$ est tel que $o o^{\prime}=o \mathrm{~A}=1$, et que la surface $o^{\prime} f^{\prime} \mathrm{A} o=\mathrm{A}_{0}^{1}=0,7803$ diffère peu de $\frac{\pi}{4}=0,78$ ว 4 ; la courbe $o f^{\prime} \mathrm{A}$ ne diffère pas beaucoup d'une circonférence. On peut représen-

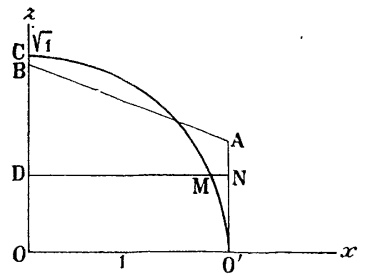

Fig. 5.

ter l'intégrale A par la moyenne arithmétique de l'aire définie par la circonférence $\mathrm{O}^{\prime} \mathrm{MC}$ de rayon:1 ( $\left.f g .5\right)$ et de l'aire définie par la ligne 
brisée $\mathrm{O}^{\prime} \mathrm{NAB}$ où $\mathrm{O}^{\prime} \mathrm{A}=0,6$ et $\mathrm{OB}=0,9$. . On a par exemple:

$$
A_{0}^{0^{\prime N}}=\frac{1}{2}[\text { aire ODMO' }+ \text { aire ODNO }]
$$

Alors :

$1^{\circ}$ Pour $0<z<0,6$ :

$$
\mathrm{A}_{0}^{\tilde{z}}=\frac{1}{4}\left[\arcsin z+z \sqrt{1-z^{2}}\right]+\frac{1}{2} z ;
$$

$2^{\circ}$ Pour $0,6<z<0,9$ :

$$
A_{0}^{z}=\frac{1}{4}\left[\arcsin z+z \sqrt{1-z^{2}}\right]+0,3+\frac{1,3-z}{1,4}(z-0,6) \cdot ;
$$

$3^{\circ}$ Pour 0,9 ๖ $<z<1$ :

$$
\mathrm{A}_{0}^{\tilde{z}}=\frac{1}{4}\left[\arcsin z+z \sqrt{1-z^{2}}\right]+0,38 \pi 5
$$

Les écarts de ces formules par rapport aux intégrales de Fresnel sont: pour les écarts maximum :

$$
+0,0031 \quad \text { et } \quad-0,0018
$$

pour l'écart absolu moyen : 0,0014.

Intégrale B. - La courbe $\eta(f g$. 3), qui présente un point d'inflexion

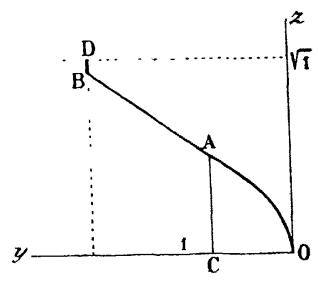

FIg. 6.

dans son premier quart de spire peut être remplacée par l'arc OA de la parabole $y=\frac{\pi}{8} z^{2}(f g .6)$ et par la droite $\mathrm{AB}$ définie par les points :
$\mathrm{A}\left[z=0,5 ; y \frac{\pi}{8}=0,3927\right]$
et
$\mathrm{B}[z=0,92 ; y=1]$. 
On a alors :

$1^{\circ}$ Si $0<z<0,5$ :

$$
\mathrm{B}=\frac{\pi}{2} \int z^{2} d z=0,5236 z^{3} ;
$$

$2^{\circ}$ Si $0, \breve{5}<z<0,92$ :

$$
\mathrm{B}=0,0654+[0,7237+0,0312](z-0, \tilde{3}) ;
$$

$3^{\circ}$ Si $0,92<z<1$ :

$$
\mathrm{B}=z-0,5621 .
$$

Pour ces formules, les écarts maximums sont:

$$
-0,0018 \text { et } \quad+0,0009 \text {; }
$$

l'écart absolu moyen est 0,00063 .

$2^{\circ}$ Second, troisieme, ..., quarts de spire. - Intégrale A. - Chaque boucle de $\xi$ est remplacée par une parabole ayant ox pour axe et ayant pour sommet le point de contact de $\zeta$ avec le cylindre (fig. 2).

L'arc de parabole se termine à son point de contact avec la tangente à la courbe $\xi$ au point de rencontre de $\xi$ avec $o z$, c'est cette tangente qui remplace alors la parabole jusqu'à l'arc de parabole suivant.

Pour les quarts de spire $\check{5}, 6, \ldots$, les arcs de parabole peuvent être remplacés par leurs cordes, et le calcul de A se fait alors par les formules (2), qui donnent:

$$
\mathrm{A}_{0}^{\tilde{z}}=\mathrm{A}_{0}^{\sqrt{n}}+\frac{2 d}{\pi}\left[\sin \frac{\pi}{2} z^{2}-\sin n \frac{\pi}{2}\right]
$$

en posant $\alpha=\frac{\pi}{2}, d=\sqrt{n+1}-\sqrt{n} ; n+1$ est le numéro d'ordre du quart de spire.

Les écarts extrêmes des valeurs obtenues pour A par rapport aux valeurs exactes sont : $-0,0016$ et $+0,0016$; l'écart absolu moyen est 0,00097 .

Intégrale B. - La courbe $\eta$ est remplacée dans le second quart de spire par une parabole dont le sommet est au point de contact de la courbe $\eta$ avec le cylindre et qui passe par l'intersection de $\eta$ avec $o z$.

Dans le troisième quart de spire, on opère comme pour $\xi$. Pour les quarts de spire suivants, on remplace encore les arcs de parabole 
par leurs cordes, et l'on a :

$$
\mathrm{B}_{0}^{z}=\mathrm{B}_{0}^{\sqrt{n}}-\frac{2 d}{\pi}\left[\cos \frac{\pi}{2} z^{2}-\cos n \frac{\pi}{2}\right]
$$

Les écarts maximum obtenus sont $+0,0024$ et $-0,0016$.

L'écart absolu moyen est 0,00078 .

V. - Intégrateur. - L'appareil construit pour le calcul des intégrales de Fresnel est représenté sur la fig. 7. Il consisle en un

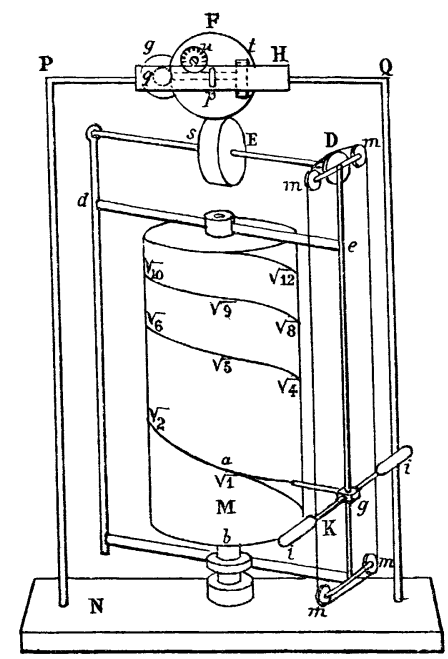

FIG. 7.

cylindre $\mathrm{M}$, fixé à demeure sur la planchette $\mathrm{N}$. Ce cylindre porte le tracé de la parabole. Pour fixer les idées, admettons que l'ordonnée $a b$, distante de $90^{\circ}$ du sommet $\mathrm{O}$ de la parabole soit égale à 10 centimètres. Ce chiffre nous doit représenter la racine carrée de l'unité conventionnelle de longueur dans nos mesures, car l'ordonnée susdite doit être égale à $\sqrt{\mathbf{1}}$. Le rayon du cylindre sera aussi de 10 centimètres. Un cylindre de 30 centimètres de longueur servira à calculer les intégrales de Fresnel pour 12 quadrants, car l'ordonnée $\sqrt{\mathbf{1 2}}$ sera égale à $\mathbf{3 4 , 6}$ centimètres. Autour de l'axe immobile du cylindre tourne librement un cadre rectangulaire cdef. Sur le côté prismatique ef glisse la coulisse $g$ qui porte l'index $h$. On suit avec cet index le tracé de la parabole au moyen des manchons ii. Au 
point $\mathrm{K}$ d'un des manchons est fixé un fil métallique sans fin qui passe par les quatre poulies $m$ et s'enroule sur la roulette $\mathrm{D}$, comme le montre la $f i g$. 8. L'axe $\mathrm{E}$ de la roulette, perpendiculaire à l'axe du cylindre et passant par le prolongement de cet axe, porte un tambour $s$ en celluloïd. Nous admettrons que le rayon de ce tambour est $2, \check{a}$ fois plus grand que celui de la roulette. Dans ce cas,

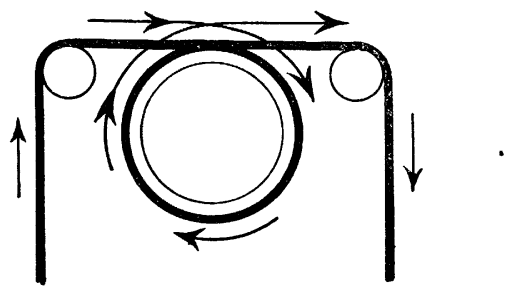

FIG. 8 .

quand l'index $h$ fera parallèlement à l'axe du cylindre une excursion égale à l'unité ( $=10$ centimètres), un point du tambour va parcourir 28 centimètres. Sur le tambour presse une boule en verre dépoli $\mathrm{F}$. La position du centre de cette boule est parfaitement déterminée par les points de contact avec trois roulettes $p, q, s$, fixées sur des axes mobiles librement dans un cadre GH ; cet axe est lié invariablement à une barre $\mathrm{PQ}$, soutenue par des colonnes, reposant sur la planchette N. La rotation du tambour $s$ produit par frottement un mouvement de rotation de la boule dans le même sens et avec une vitesse linéaire identique. L'axe de la roulette $p$ est parallèle au diamètre du cylindre passant par le sommet $\mathrm{O}$ de la parabole; l'axe de la roulette $q$ est perpendiculaire à cette direction. La position initiale du cadre portant l'index est telle que l'index coïncide alors avec le sommet de la parabole, et l'axe E est parallèle à l'axe de la roulette $p$. Si nous relevons l'index en suivant la direction du cylindre, c'est-à-dire sans tourner le cadre $c$ def, la roulette $p$ prend un mouvement de rotation, tandis que la roulette $g$ reste en repos. Une excursion de l'index égale à l'unité conventionnelle fait marcher un point de la boule de 25 centimètres. Si la circonférence de la poulie est égale à 20 millimètres, elle fait 10 tours par unité de l'excursion de l'index. L'axe de la poulie $p$ porte une tête cylindrique $t$ divisée en 100 parties égales, dont les dixièmes peuvent être encore appréciés. Chaque tour de la roulette est enregistré par l'intermédiaire d'une vis sur le disque $u$. Ainsi l'ordonnée unité est représentée par 1000 divisions 
de la tête ou par 10.000 dixièmes d'une division. Bien entendu, en faisant croitre les dimensions de l'appareil, on peut faire croître la précision de la lecture. Admettons maintenant que le cadre portant l'index ait tourné de l'angle $v$. Alors (comme le montre la $f g .9$ ) une excursion de l'index égale à $d y$ fait tourner la roulette $p$ de la quantité $d y \cos v$, tandis que la roulette $q$ tourne de $d y \sin v$. Ainsi le

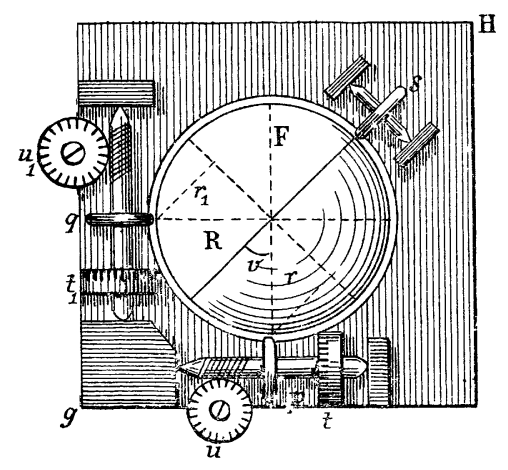

FIG. 9.

disque $u$ et le tambour $t$ enregistrent les valeurs des intégrales A; et le disque $u_{1}$ et le tambour $t_{1}$, les intégrales B. Pour cela, il suffit de soustraire les deux lectures initiales sur les deux appareils enregistreurs des deux lectures finales et de diviser le résultat par 10000 .

La construction des parties supérieures de l'intégrateur rappelle celle des enregistreurs de l'analyseur harmonique, construit par M. Coradi, pour développer une fonction en série de Fourier. Les précautions qu'il faut prendre en faisant des mesures sont les mêmes pour cet appareil et pour l'intégrateur, aussi sera-t-il utile de consulter, pour plus de détails, les deux mémoires suivants: G. Coradi, Der Harmonische Analysator; Zürich, 1894; - et N. Bervy, Sur un analyseur harmonique (Annales de l'Observatoire de Moscou). 